\title{
How does Surface Tension affect Energy Release Rate of Cracks loaded in Mode I ?
}

\author{
Chung-Yuen Hui ${ }^{*}$, Tianshu Liư ${ }^{1}$, Marie-Emeline Schwaab² \\ ${ }^{1}$ Field of Theoretical and Applied Mechanics, Department of Mechanical and Aerospace Engineering, \\ Cornell University, Ithaca, NY 14853, USA \\ ${ }^{2}$ Institut de Recherche en Génie Civil et Mécanique (GeM), UMR CNRS 6183, Ecole Centrale de Nantes, BP \\ 92101,44321 Nantes cedex 3, France \\ *Corresponding Author, ch45@cornell.edu
}

\begin{abstract}
The role of surface stress on fracture of elastic solids has been studied by two group of researchers with different conclusions. One group concludes that surface stress has no effect on energy release rate except that the singularity of the crack tip field is stronger than the usual inverse square root singularity dictated by linear elastic fracture mechanics. The other group concludes that the singularity of the stress field reduces to a logarithmic singularity, thus implying that the local energy release rate is zero. In this letter we resolve this paradox by examining the solution of a special case where surface stress is isotropic and independent of surface strain. We show that surface tension resists crack growth by lowering the applied energy release rate while retaining the inversely square root singularity of the elastic crack tip field. We demonstrate this idea by solving a perturbation problem where the capillary length is much smaller than the crack length. A closed form expression for the local energy release rate is obtained in the limit of small surface tension.
\end{abstract}

Keywords: surface tension; crack; energy release rate; stress intensity factor; Laplace pressure

\section{Introduction}

The characteristic length scale that controls the deformation of a solid due to its surface tension $\sigma$ is given by the ratio of the surface tension to its shear modulus $G$. For hard materials such as metals and ceramics, this "capillary" length $\sigma / G$ is smaller than atomic dimensions, hence surface tension effect can be ignored. However, the capillary length of soft materials such as hydrogels and elastomers range from tens of $\mathrm{nm}$ to hundreds of $\mu \mathrm{m}$. For this class of materials, surface tension can drive shape change, for example, a sharp corner in a soft material cannot remain sharp because of surface tension ${ }^{1,2}$. Surface tension can also flatten surfaces of structures made by replica molding ${ }^{3,4}$ and cause Plateau instability in thin gel filaments ${ }^{5}$. However, surface tension can resist deformation, for example, both experiments and theory has shown that the contact mechanics of spheres and cylinders on soft elastic substrates can be affected by solid surface tension ${ }^{6-11}$. Liu et al. ${ }^{12}$ have shown that surface tension induced Laplace pressure can cause closure of a crack inflated by hydrostatic pressure.

There are very few experimental studies examining the role of surface stress in fracture since actual measurements of surface stresses are very difficult to make. The first theoretical study on the influence of surface stress on the fracture of elastic solids were carried out by Thomson, Chuang and $\mathrm{Lin}^{13}$ in 1986 (hence forth referred as TCL). In their model, they ignored the curvature of the deformed crack faces, and the effect of surface stresses is modelled as a line 
force acting at the crack tip. Their analysis showed that even though the singularities due to the line force are much higher than the typical inverse square root singularity in linear elastic fracture mechanics, they have no effect on the crack tip energy release rate [see remarks after eq. 42 in their paper].

It is interesting to compare TCL's result with the more recent studies of Kim, Schiavone and $\mathrm{Ru}^{14,15}$ (hence forth refer as KSR). Using the surface constitutive model of Gurtin and Murdoch ${ }^{16}, \mathrm{KSR}^{14}$ in 2010 formulated a small strain theory of fracture to study the effect of surface tension on fracture of a plane strain crack loaded in Mode I and Mode II. Their numerical results showed that the stress field directly ahead of a Mode I crack is bounded, irrespective of the magnitude of the surface stress (as long as it is not exactly zero). In a later paper $^{14}$, KSR reexamined their numerical solution in greater details and concluded that the stress field directly ahead of the crack tip has a weaker logarithmic singularity. In both cases their numerical results imply that the stress intensity factor (or the local stress intensity factor $K_{\text {local }}=K_{A}+K_{S T}$ ) is exactly zero. In other words, the applied stress intensity factor $K_{A}$ is cancelled by the negative stress intensity factor $K_{S T}$ caused by the curvature induced Laplace pressure acting to close the crack faces. Since a zero local stress intensity factor implies that local energy release rate is also zero, their result is the exact opposite of TCL's. Furthermore, because surface tension is represented as a line force, the stress field near the crack tip in TCL model is more singular than the inverse square root singularity of the classical theory and this result also contradicts the result of KSR.

The goal of this letter is to resolve this paradox. To simplify the analysis, we focus on Mode I cracks and the surface stress $\sigma_{\alpha \beta}$ is assumed to be isotropic and is a constant independent of surface strain, that is, $\sigma=\sigma \boldsymbol{I}$ where $\sigma$ is the surface tension (force/length). To focus attention on the crack tip, we consider the "Small Scale Surface tension" problem in which the effect of surface tension is confined to a region that is small with respect to typical specimen dimensions. As in Small Scale Yielding in classical fracture theory ${ }^{17}$, the crack is semi-infinite and lies on the negative $x$ axis, as shown in Figure 1 . The boundary condition is that at distances far from the crack tip, the stress and deformation field approaches the usual inverse square root singularity of the classical elasticity solution. More details will be given in the next section.

The curvature induced Laplace pressure $p_{L}$ resisting the opening of the crack (see Fig. 1 ) is related to the surface tension $\sigma$ and the curvature of the deformed crack faces $\kappa$ by:

$$
p_{L}=\sigma \kappa \quad \kappa=\frac{\mathrm{v}^{\prime \prime}}{\left[1+\left(\mathrm{v}^{\prime}\right)^{2}\right]^{3 / 2}}
$$

where $v$ is the crack opening displacement and a prime denotes differentiation with respect to $\mathrm{x}$. In KSR, $\mathrm{v}^{\prime}$ is assumed to be small everywhere and $\kappa$ is approximated by:

$$
\kappa=\mathrm{v}^{\prime \prime}
$$

Due to this approximation, the governing equation describing the crack tip field is linear and the full machinery of analytic function theory can be used to formulate the crack problem. In 
contrast, TCL avoided this issue all together by placing a line force at the crack tip - the curvature induced Laplace pressure appears as a delta function in their formulation ${ }^{12}$.
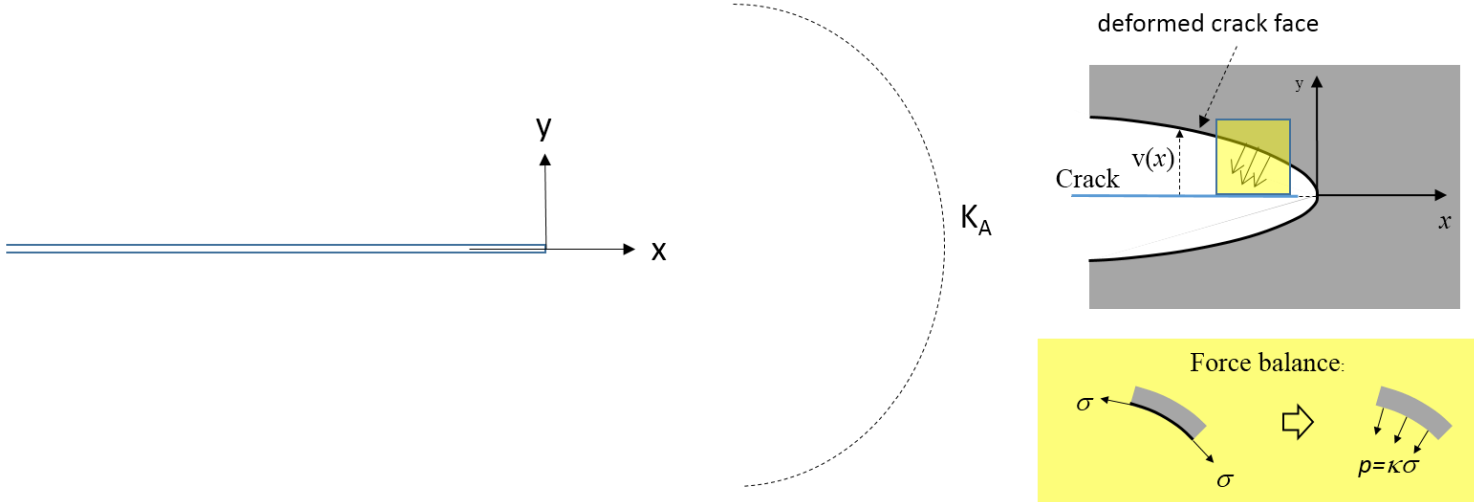

Figure 1 Figure on left is a schematic of the geometry, where an undeformed semi-infinite plane strain Mode I crack occupying the negative $x$ axis is subjected to a remote far field controlled by the applied stress intensity factor $K_{A}$. Figure on right shows the deformed crack face, which is subjected to a Laplace pressure induced by local curvature of the deformed crack surface.

It is easy to understand why $\mathrm{K}_{\text {local }}$ had to be zero in KSR's analysis, that is, the local stress field near the crack tip cannot have an inverse square root singularity. Indeed, assuming $\mathrm{K}_{\text {local }}$ is positive, the crack opening for the upper crack face near the crack tip is:

$$
\mathrm{v}=\frac{2(1-v) K_{\text {local }}}{G \sqrt{2 \pi}} \sqrt{-x}, \quad x<0
$$

where $v$ is the Poisson's ratio and $G$ the shear modulus. Substituting (3) into $\kappa=v^{\prime \prime}$ gives

$$
\kappa \propto-K_{\text {local }}|x|^{-3 / 2}, \quad x \rightarrow 0^{-}
$$

Equations (4) and (1) imply that the Laplace pressure has a non-integrable singularity; such a singular pressure field will induce an infinite negative stress intensity factor at the crack tip, which means that $K_{\text {local }}$ goes to negative infinity. This is a contradiction to the original assumption that $K_{\text {local }}$ is positive so the only possibility is $K_{\text {local }}=0$, which implies that the stress is bounded or has a weaker singularity.

The fact that $K_{\text {local }}=0$ brings up the possibility a line force can exist at the crack tip. Indeed, in linear elasticity fracture mechanics, the crack tip deforms into a cusp shape if the stress is bounded at the tip - the Dugdale-Barenblatt model being a prime example ${ }^{17-19}$; whereas a logarithmic singularity in stress implies the crack tip opens into a wedge [see Appendix]. In both cases the curvature has a delta function behavior which corresponds to existence of a line force acting at the crack tip. Thus, the crack tip stress field in the KSR model can still be singular since the singularity in curvature can causes a singular field similar to those studied by TCL. 
Specifically, an implicit assumption of KSR is that curvature is non-singular; otherwise a concentrated line force can act at the crack tip.

The key idea in this letter is that these difficulties can be resolved using the full curvature expression. To see this, let us assume that the local crack tip field has form given by standard theory (3), then (1) implies that

$$
\kappa\left(x \rightarrow 0^{-}\right) \rightarrow \frac{\mathrm{v}^{\prime \prime}}{\left|\mathrm{v}^{\prime}\right|^{3}}=\frac{-\pi G^{2}}{2(1-v)^{2} K_{\text {local }}^{2}}
$$

The key feature is that $\mathrm{v}^{\prime \prime}$ and $\left(\mathrm{v}^{\prime}\right)^{3}$ has exactly the same singularity, so the curvature is bounded at the crack tip. This result implies that the curvature induced Laplace pressure is finite everywhere along the crack face including the crack tip. Thus, for sufficiently small surface tensions, the local stress intensity factor is well defined and positive (hence there is a non-zero energy release rate). In addition, the existence of finite curvature eliminates the possibility of a singular line force acting at the crack tip.

\section{Formulation and Analysis}

To quantify our idea, we employed the usual small scale yielding formulation where the crack is modeled as semi-infinite and lies on the negative $x$ axis with its tip at $x=0$. For our case, the plastic zone is replaced by a zone where surface curvature is significant. This formulation is valid in the limit where the capillary length $\sigma / G$ is much smaller than the crack length $a$. For example, the shear modulus of a soft hydrogel is on the order of 100 to $1000 \mathrm{~Pa}$, using a crack length of $1 \mathrm{~cm}, \sigma / \mathrm{Ga}$ ranges from 0.07 to 0.007 , where we have taken the surface tension of the hydrogel to be equal to the surface tension of water, which is about $0.07 \mathrm{~N} / \mathrm{m}$. Thus, for most soft materials, the region of dominance of surface tension is expected to be localized at the crack tip. Let $K_{A}$ denote the applied stress intensity factor. It is the stress intensity factor in the absence of surface tension. The displacement of the upper crack face $v_{A}$ due to the applied $K_{A}$ field is

$$
v_{A}=\frac{2(1-v) K_{A}}{G} \sqrt{-x / 2 \pi} \quad x<0
$$

Surface tension $\sigma$ caused a restraining pressure $p_{L}(x)$ to close the crack, see (1). The crack face displacement $v_{p}$ due to an arbitrary pressure field $p$ acting on the crack faces is well known ${ }^{20}$; setting $p=p_{L}(x)$ gives:

$$
v_{p}=\frac{(1-v)}{G \pi} \int_{-\infty}^{0} p_{L}(t) \ln \mid \frac{\sqrt{|x|}+\sqrt{|t|} \mid}{\sqrt{|x|}-\sqrt{|t|} \mid} d t
$$


Note that since the Laplace pressure is negative, $v_{p}<0$. The actual crack opening displacement is the sum of (5) and (6)

$$
v=v_{A}+v_{p}
$$

The Laplace pressure is related to the curvature of the crack opening displacement $v$ by (1). Substituting this into (7-8), (8) becomes:

$$
v(x)=\frac{2(1-v) K_{A}}{G} \sqrt{-x / 2 \pi}+\frac{(1-v) \sigma}{\pi G} \int_{-\infty}^{0} \frac{v^{\prime \prime}(t)}{\left[1+\left(v^{\prime}(t)\right)^{2}\right]^{3 / 2}} \ln \left(\frac{\sqrt{-x}+\sqrt{-t}}{\sqrt{-x}-\sqrt{-t}}\right) d t \quad x<0
$$

For a given applied stress intensity factor, (9) is a non-linear integral equation for the unknown crack opening displacement $v$. Equation (8) can be reduced to a more standard form by differentiating both sides by $x$, resulting in:

$$
v^{\prime}(x)=-\frac{(1-v) K_{A}}{G} \sqrt{\frac{-1}{2 \pi x}}+\frac{(1-v) \sigma}{\pi G \sqrt{-x}} P V\left[\int_{-\infty}^{0} \frac{\sqrt{-t}}{t-x} \frac{v^{\prime \prime}(t)}{\left[1+\left(v^{\prime}(t)\right)^{2}\right]^{3 / 2}} d t\right] \quad x<0
$$

where $P V$ denotes principal value. More insight can be obtained by normalizing $x$ by the elasto-capillary length and the opening displacement $v$ by the opening displacement of a traction free crack evaluated at a distance equal to the elasto-capillary length behind the crack tip, i.e.,

$$
\eta=\frac{\pi G}{(1-v) \sigma} x, \quad v=\frac{2(1-v) K_{A}}{G} \sqrt{\frac{(1-v) \sigma}{2 \pi^{2} G}} \bar{v}
$$

Using these normalized variables, the governing equation (9) becomes:

$$
\dot{\bar{v}}(\eta)=\frac{-1}{2 \sqrt{-\eta}}+\frac{1}{\sqrt{-\eta}} \int_{-\infty}^{0} \frac{\sqrt{-\xi}}{\xi-\eta} \frac{\ddot{\bar{v}}(\xi)}{\left[1+\beta(\dot{\bar{v}}(\xi))^{2}\right]^{3 / 2}} d \xi \quad \eta<0
$$

where a dot denote differentiation with respect to the normalized distance $\eta$ and

$$
\beta \equiv \frac{2(1-v) K_{A}^{2}}{G \sigma}=4\left(J_{A} / \sigma\right)
$$

is a dimensionless parameter. It is proportional to the ratio of the energy release rate without surface tension, $J_{A}$, to the surface tension. It is particular interesting to note that if the approximation $\kappa \approx v^{\prime \prime}$ is used, (12a) becomes 


$$
\dot{\bar{v}}(\eta)=\frac{-1}{2 \sqrt{-\eta}}+\frac{1}{\sqrt{-\eta}} \int_{-\infty}^{0} \frac{\sqrt{-\xi}}{\xi-\eta} \ddot{\bar{v}}(\xi) d \xi \quad \eta<0 .
$$

Note that there is no free parameter in (13). The absence of $\beta$ means that the near tip fields is universal, that is, increasing or decreasing the surface tension could only change the region of asymptotic validity of the near tip fields, but not its form. Our asymptotic analysis of (13) supports the numerical results of $\mathrm{KSR}^{14}$ - the normal stress $\sigma_{y y}$ directly ahead of the crack tip has a logarithmic singularity. This analysis is given in the appendix for completeness.

The local stress intensity factor $K_{\text {local }}$ can be expressed by adding the applied stress intensity factor to the stress intensity factor induced by the Laplace pressure. This can be done by examining the behavior of $v^{\prime}\left(x \rightarrow 0^{+}\right)$in (9) or by using a standard expression in fracture mechanics ${ }^{20}$ :

$$
K_{\text {local }}=K_{A}+\sigma \sqrt{\frac{2}{\pi}} \int_{-\infty}^{0} \frac{v^{\prime \prime}(t)}{\left[1+v^{\prime}(t)\right]^{3 / 2}} \frac{d t}{\sqrt{-t}}
$$

In normalized form, (14) is

$$
\frac{K_{\text {local }}}{K_{A}}=1+\chi(\beta), \quad \chi(\beta)=2 \int_{-\infty}^{0} \frac{\ddot{\bar{v}}(\xi)}{\left(1+\beta \dot{\bar{v}}^{2}(\xi)\right)^{3 / 2}} \frac{d \xi}{\sqrt{-\xi}}
$$

Note that $\chi(\beta)$ is in general negative $(\ddot{\bar{V}}(\xi)<0)$ there is a reduction in stress intensity factor. In this letter we limit our study to small surface tension where $\beta>>1$. In this limit, the integral term in (12a) is small compare with the first term so that

$$
\dot{\bar{v}}(\eta)=\frac{-1}{2 \sqrt{-\eta}}+O(1 / \beta)
$$

Therefore a first order estimate of $K_{\text {local }}$ can be determined by substituting $\dot{\bar{v}}(\eta) \approx \frac{-1}{2 \sqrt{-\eta}}$ in $(16)$ into (15) and evaluating $\chi(\beta)$. The resulting integral can be evaluated exactly and we found

$$
\frac{K_{\text {local }}}{K_{A}}=1-\frac{4}{\beta} . \quad \quad \beta>>1
$$

Since the energy release rate is proportional to the square of the stress intensity factor, (17) is equivalent to

$$
\frac{J_{\text {local }}}{J_{A}}=\left(1-\frac{4}{\beta}\right)^{2} . \quad \quad \beta>>1
$$


where $J_{\text {local }}$ is the energy release rate at the crack tip. Equation (18) indicates that surface tension reduces applied energy release rate gradually and smoothly.

\section{Discussion}

Our model gives a satisfactory resolution of the differences in result between the work of TCL and KSR. To summarize, in TCL, the curvature of the deformed crack faces are ignored, the surface tension acts on the un-deformed flat crack faces. The line force boundary condition corresponds to the sudden change of slope at the crack tip. In contrast, KSR imposed a closing pressure on the undeformed crack faces which is proportional to the curvature of the deformed crack faces. These two different approaches gave very different results: in $\mathrm{TCL}$, surface tension has no effect on the applied energy release rate, but increases the stress singularity at the crack tip; while for KSR, surface tension eliminates the local energy release rate by reducing the stress singularity at the crack tip.

Our approach is to treat the surface tension induced Laplace pressure as a cohesive traction acting on the crack faces. Cohesive traction (force) model was first introduced by Barenblatt ${ }^{19}$ to study brittle fracture. His idea is that the separation of perspective fracture surfaces directly ahead of a crack tip is resisted by atomic cohesive forces. Since its introduction cohesive force model has been used to study a wide variety of fracture phenomenon. In the standard theory, the cohesive traction is a nonlinear function of crack opening displacement. For our case, the cohesive traction depend only on the curvature of the crack face (or the first and second derivatives of the crack opening displacement). Our model is mathematically equivalent to KSR, except we retain the nonlinear terms in the evaluation of curvature. Retaining this nonlinear term smooth over the curvature field and avoids the dominance of surface tension over elasticity near the crack tip. The result is that for small surface tensions or large $\beta$, the local stress field has the same square root singularity, but with a reduced stress intensity factor. The applied energy release rate is also reduced by surface tension. Of course, if $\beta$ is small, that is, if the surface tension is large or the applied stress intensity factor small, then the Laplace pressure could close the crack and eliminate the inverse square singularity at the crack tip. We did not study this scenario, but a recent paper by Liu et $\mathrm{al}^{12}$ showed that the local energy release of an internally pressurized crack subjected to large deformation can vanish or even become negative for sufficiently large surface tensions or small internal pressures.

Our approach can be readily applied to finite cracks in elastic solids, as long as the Green's function for line load acting on the crack faces is known. Consider for example a plane strain crack of length $2 \mathrm{a}$ in an infinite elastic block subjected to remote tension $\sigma_{A}$. For this case, the crack opening displacement due to the Laplace pressure $v_{p}$ is related to the actual crack opening displacement $v$ by

$$
v_{p}=\frac{(1-v) \sigma}{G \pi} \int_{-a}^{a} \frac{v^{\prime \prime}(t)}{\left[1+\left(v^{\prime}(t)\right)^{2}\right]^{3 / 2}} \ln \left|\frac{\sqrt{a^{2}-x^{2}}+\sqrt{a^{2}-t^{2}}}{\sqrt{a^{2}-x^{2}}-\sqrt{a^{2}-t^{2}}}\right| d t
$$


where we have used a standard result ${ }^{20}$. The crack opening displacement $v_{A}$ due to the applied remote tension $\Sigma_{A}$ is

$$
v_{A}=\frac{(1-v) \Sigma_{A}}{G} \sqrt{a^{2}-x^{2}}
$$

The integral equation governing the unknown $v$ is obtained by combining (18-19):

$$
v(x)=\frac{(1-v) \Sigma_{A}}{G} \sqrt{a^{2}-x^{2}}+\frac{(1-v) \sigma}{G \pi} \int_{-a}^{a} \frac{v^{\prime \prime}(t)}{\left[1+\left(v^{\prime}(t)\right)^{2}\right]^{3 / 2}} \ln \left|\frac{\sqrt{a^{2}-x^{2}}+\sqrt{a^{2}-t^{2}}}{\sqrt{a^{2}-x^{2}}-\sqrt{a^{2}-t^{2}}}\right| d t \quad|x|<a \quad \text { (20) }
$$

There is obvious limitation to the small strain approach used in this work, especially for very soft solids where the deformation can be exceeding large near the crack tip. An example of a crack subjected to very large deformation can be found in Sun et al. $^{21}$. The fact that we need to retain the nonlinear term in curvature suggests that large deformation matters. Therefore, a consistent approach is to carry out a fully nonlinear large deformation analysis such as those recently performed by Liu et al 12. The stress free reference configuration for a cracked elastic solid can be obtained by imagining turning off surface tension (e.g. treating the crack surfaces with a self-assembled monolayer) in the absence of external load. Starting from this stress free configuration, imagine turning on the surface tension without applying any external load (removing the monolayer). This sudden engagement of surface tension will immediately give a very concentrated line force at the crack tip. This line force will pull the crack tip inwards and resulting in the opening the crack faces. Since the surface stresses depend on the deformed configuration, the line force will be smooth over by the deformation, the curvature of the crack faces is expected to be continuous. This picture is consistent with Liu et al's simulation of a penny-shape crack in a neo-Hookean solid ${ }^{12}$.

Finally, we mention that the reduction of energy release rate in our analysis is consistent with the earlier analysis of $\mathrm{Wu}^{22}$ where an artificial finite tip radius was introduced to avoid the singularity of curvature. This finite tip radius approach was also used in later works by Fu et al. ${ }^{23}$ and Wang et al. ${ }^{24}$

\section{Acknowledgement}

C.Y. Hui and T. Liu acknowledge support from the U.S. Department of Energy, Office of Basic Energy Science, Division of Material Sciences and Engineering under Award (DE-FG02-07ER46463). MarieEmeline Schwaab was a student from Ecole Centrale de Nantes who participated in this research during her visit to Hui's group at Cornell. Her visit and work was supported by the Région Pays de la Loire. C.Y. Hui enjoyed the many discussions with Anand Jagota and Rong Long.

\section{References}

1 C.-Y. Hui, A. Jagota, Y.Y. Lin and E.J. Kramer,Langmuir, 2002, 18 , 1394

2 S. Mora. and Y. Pomeau, Condens. Matter, 2015, 27, 194112

3 A. Jagota, D. Paretkar, and A. Ghatak, Phys. Rev. E , 2012, 85, 051602

4 D. Paretkar, X. Xu, C.Y. Hui and A. Jagota, Soft Matter, 2014, 10, 4084

5 S. Mora, T. Phou, J. Fromental, L. Pismen, and Y. Pomeau, Phys. Rev. Lett.,2010, 105,214301 
R.W. Style, C. Hyland, R. Boltyanskiy, J.S. Wettlaufer, and E.R. Dufresne, Nat. Commun., 2013, 4, 2728

$7 \quad$ J.-M.Y. Carrillo and A. V Dobrynin, Langmuir, 2012, 28, 10881.

$8 \quad$ Z. Cao, M.J. Stevens, and A. V. Dobrynin, Macromolecules, 2014, 47, 6515.

9 T. Salez, M. Benzaquen, and É. Raphaël, Soft Matter, 2013, 9, 10699.

10 X. Xu, A. Jagota, and C.-Y. Hui, Soft Matter, 2014, 10, 4625.

11 C.-Y. Hui, T. Liu, T. Salez, E. Raphael, and A. Jagota, Proc.R.Soc.A, 2014, 471: 20140727.

12 T. Liu,R. Long and C.Y. Hui, Soft matter, 2014, 10, 7723

13 T. Thomson, T.-J. Chuang, I.-H. Lin, Acta metall. 1986, 34, 1143

14 C.I. Kim, P. Schiavone and C.Q. Ru, J. Elasticity, 2010, 104, 397

15 C.I. Kim, C.Q. Ru and P. Schiavone, Math. \& Mech. of Solids, 2012, 18, 59

16 M.E. Gurtin and A.I. Murdoch, Arch. Rational Mech. Anal, 1975, 54, 291

17 J. R. Rice, "Mathematical Analysis in the Mechanics of Fracture", Chapter 3 of Fracture: An

Advanced Treatise (Vol. 2, Mathematical Fundamentals) (ed. H. Liebowitz), Academic Press, N.Y., 1968, pp. 191-311.

18 D.C. Dugdale, J. Mech. Phys. Solids, 1960, 8, 100

19 G.I. Barenblatt, Adv. Appl. Mech., 1962, 7, 55

20 H. Tada, P. C. Paris and G.R. Irwin, "The stress analysis of cracks Handbook", $3^{\text {rd }}$ edition, p87. ASME publication (2000), ISBN 10: 0791801535

21 J.Y. Sun, X. Zhao, W. R. Illeperuma, O. Chaudhuri, O; K.H. Oh, D.J. Mooney, J.J. and Z. Suo, Nature, 2012, 489, 133.

22 N.I. Muskhelishvili, "Singular Integral Equations", Chapter 4, $2^{\text {nd }}$ Edition, 1992, Dover

Publications, INC. New York

23 C. Wu, J. Mech. Phys. Solids, 1999, 47, 2469

24 G.-F. Wang, X.-Q. Feng, T.-J. Wang and W. Gao, J. Appl. Mech. 2008, 75, 011001 


\section{Appendix: Asymptotic behavior of (13)}

Let $f(\eta)=\dot{v}(\eta)$, then (13) can be rewritten as:

$f(\eta)=\frac{-1}{2 \sqrt{-\eta}}-\frac{1}{\sqrt{-\eta}} \int_{-\infty}^{0} \frac{\sqrt{-\xi} \dot{f}(\xi)}{\xi-\eta} d \xi$

Define

$$
\phi(z) \equiv \frac{1}{2 \pi i} \int_{-\infty}^{0} \frac{\sqrt{-\xi} \dot{f}(\xi)}{\xi-z} d \xi \quad z \notin(-\infty, 0]
$$

where $z=\eta+i \zeta$ is a complex variable. The function $\phi$ is analytic in the entire complex $z$ plane with the exception of the cut $(-\infty, 0]$. Using the Plemelj formulae, we have, for $\eta \in(-\infty, 0)$,

$$
\begin{aligned}
& \phi^{+}(\eta)-\phi^{-}(\eta)=\sqrt{-\eta} \dot{f}(\eta) \\
& \phi^{+}(\eta)+\phi^{-}(\eta)=\frac{1}{\pi i} \int_{-\infty}^{0} \frac{\sqrt{-\xi} \dot{f}(\xi)}{\xi-\eta} d \xi
\end{aligned}
$$

where the superscripts \pm denote the values of $\phi$ as $z$ approaches the cut $(-\infty, 0]$ from above or below respectively. Also, the integral in ( $\mathrm{A} 3 \mathrm{~b})$ is interpreted as a principal value integral. The asymptotic analysis is easiest to carry out if we formulate $A 1$ as a functional equation, as shown below. Equation (A3b) implies that

$$
\pi i\left[\phi^{+}(\eta)+\phi^{-}(\eta)\right]=\int_{-\infty}^{0} \frac{\sqrt{-\xi} \dot{f}(\xi)}{\xi-\eta} d \xi
$$

Substituting (A4) into (A1), (A1) becomes,

$$
f(\eta)=\frac{-1}{2 \sqrt{-\eta}}-\frac{\pi i}{\sqrt{-\eta}}\left[\phi^{+}(\eta)+\phi^{-}(\eta)\right] \quad \eta \in(-\infty, 0)
$$

Now differentiate $A 5$ with respect to $\eta$, it becomes

$$
\dot{f}(\eta)=\frac{-1}{4(-\eta)^{3 / 2}}-\frac{\pi i}{2(-\eta)^{3 / 2}}\left[\phi^{+}(\eta)+\phi^{-}(\eta)\right]-\frac{\pi i}{(-\eta)^{1 / 2}} \frac{d\left[\phi^{+}(\eta)+\phi^{-}(\eta)\right]}{d \eta}
$$

Substituting (A6) into the LHS of (A3a), we have 


$$
\begin{aligned}
& \phi^{+}(\eta)-\phi^{-}(\eta)=\sqrt{-\eta}\left\{\frac{-1}{4(-\eta)^{3 / 2}}-\frac{\pi i}{2(-\eta)^{3 / 2}}\left[\phi^{+}(\eta)+\phi^{-}(\eta)\right]-\frac{\pi i}{(-\eta)^{1 / 2}} \frac{d\left[\phi^{+}(\eta)+\phi^{-}(\eta)\right]}{d \eta}\right\} \\
& =\frac{1}{4 \eta}+\frac{\pi i}{2 \eta}\left[\phi^{+}(\eta)+\phi^{-}(\eta)\right]-\pi i \frac{d\left[\phi^{+}(\eta)+\phi^{-}(\eta)\right]}{d \eta}
\end{aligned}
$$

The form of (A7) suggest that as $\eta \rightarrow 0^{-}$, the RHS of (A7) should dominate the LHS, so that the first order asymptotic behavior of $\phi$ can be examined by studying the equation

$$
\frac{1}{4 \eta}+\frac{\pi i}{2 \eta}\left[\phi^{+}(\eta)+\phi^{-}(\eta)\right]-\pi i \frac{d\left[\phi^{+}(\eta)+\phi^{-}(\eta)\right]}{d \eta}=0 \quad \eta \rightarrow 0^{-}
$$

Let $g(\eta) \equiv\left[\phi^{+}(\eta)+\phi^{-}(\eta)\right]$, then (A7) implies that

$$
\frac{d g}{d \eta}-\frac{g}{2 \eta}=\frac{1}{4 \pi i \eta} \quad \eta \rightarrow 0^{-}
$$

The general solution of (A8) is

$$
g(\eta) \equiv\left[\phi^{+}(\eta)+\phi^{-}(\eta)\right]=-\frac{1}{2 \pi i}+i A(-\eta)^{1 / 2}
$$

where $A$ is an arbitrary real constant (this is because $g$ is purely imaginary according to (A3b)). Substituting (A10) into (A5), we have

$$
f(\eta)=\frac{-1}{2 \sqrt{-\eta}}-\frac{\pi i}{\sqrt{-\eta}}\left[-\frac{1}{2 \pi i}+i A(-\eta)^{1 / 2}\right]=\pi A
$$

Thus, the asymptotic behavior of $f$ as $\eta \rightarrow 0^{-}$is a constant, and since

$$
f(\eta)=\dot{\bar{v}}(\eta) \Rightarrow \bar{v}(\eta)=\pi A \eta \quad \eta \rightarrow 0^{-}
$$

For non-zero negative $A$, the local shape of crack tip is a wedge. (A12) implies that the $\sigma_{y y}(x)$ has a logarithmic singularity. For consistency, we should check that the LHS of (A7) is indeed small in comparison with terms of the RHS as $\eta \rightarrow 0^{-}$. To do this, we solve (A10) and found

$$
\phi=-\frac{1}{4 \pi i}-\frac{i A}{2 \pi} \sqrt{z} \ln (z)
$$


To check that (A13) indeed satisfies (A6), we note that

$$
\begin{aligned}
& \phi^{+}=-\frac{1}{4 \pi i}-\frac{i A}{2 \pi} \sqrt{-\eta} i[\ln (-\eta)+\pi i] \\
& \phi^{-}=-\frac{1}{4 \pi i}-\frac{i A}{2 \pi} \sqrt{-\eta}(-i)[\ln (-\eta)-\pi i]
\end{aligned}
$$

Therefore, $\phi^{+}(\eta)+\phi^{-}(\eta)=-\frac{1}{2 \pi i}+i A \sqrt{-\eta}$. From (3a) and (A14), we found

$$
\phi^{+}(\eta)-\phi^{-}(\eta)=\frac{A}{\pi} \sqrt{-\eta} \ln (-\eta) \quad \text { as } \eta \rightarrow 0^{-}
$$

which means the term on the LHS of (A7) can be neglected compare with any term on the RHS of (A7).

Our result (A12) shows that $\dot{\bar{v}}(\eta)$ is a constant. We now show that $\bar{\sigma}_{y y}(\eta)$ as $\eta$ approach the crack tip at $\eta=0$ has a logarithmic singularity. To see this, recall that any Mode I crack (with arbitrary normal traction on its faces) can be viewed as a continuous distribution of edge dislocations ${ }^{17}$; in particular, for an semi-infinite crack lying on the negative $x$ axis,

$$
\sigma_{y y}(x)=\frac{G}{2 \pi(1-v)} \int_{-\infty}^{0} \frac{\mu\left(x^{\prime}\right)}{x-x^{\prime}} d x^{\prime} \quad x \neq 0
$$

where $\mu\left(x^{\prime}\right)=-2 d v / d x^{\prime}$ is the dislocation density. Using (11), the normalized form of (A16) is:

$$
\bar{\sigma}_{y y}(\eta)=\frac{1}{2 \pi} \int_{-\infty}^{0} \frac{\bar{\mu}(\xi)}{\eta-\xi} d \xi
$$

where $\bar{\sigma}_{y y}$ and $\bar{\mu}$ are the normalized stress and dislocation density defined by

$$
\sigma_{y y}=\frac{G \sqrt{\beta}}{\pi(1-v)} \bar{\sigma}_{y y} \quad \bar{\mu}(\xi)=-2 \dot{\bar{v}}(\xi)=-\frac{\mu}{\sqrt{\beta}}
$$

respectively. The asymptotic behavior of $\bar{\sigma}_{y y}$ as $\eta$ approach the crack tip $(\eta=0)$ can be found using a result of Muskhelishvili ${ }^{22}$ where he showed that for $\dot{\bar{v}}\left(\eta \rightarrow 0^{-}\right) \rightarrow \pi A$,

$$
\bar{\sigma}_{y y}\left(\eta \rightarrow 0^{+}\right)=\pi A l n \eta
$$

This result is consistent with a numerical finding of $\mathrm{KSR}^{15}$. 